\title{
Clinical Results of Thoracic Aortic Surgery in Patients under Hemodialysis
}

\author{
Makoto Hibino*, Akihiko Usui, Hideki Oshima, Yuji Narita, Tomonobu Abe, Yoshimori Araki, Masato Mutsuga, \\ Kazuro Fujimoto \\ From World Society of Cardiothoracic Surgeons 25th Anniversary Congress, Edinburgh \\ Edinburgh, UK. 19-22 September 2015
}

\section{Background/Introduction}

The number of patients receiving hemodialysis is increasing year by year, and the number of cases who underwent thoracic aortic surgery is limited but also increases.

\section{Aims/Objectives}

In this study, we clarified the early and mid-term results of open surgery for thoracic aortic aneurysm (TTA) in patients under hemodialysis.

\section{Method}

In consecutive 700 open surgical repair for TTA between January 2002 and October 2014, there are 21 patients under hemodialysis preoperatively. They underwent open repair for aortic root in 2, ascending in 6 , arch in 10, descending in 2 and thoracoabdominal aorta in 1 . They were 20 male and 1 female with mean age of $63.3 \pm 13.3$ years and mean duration of hemodialysis was $3.4 \pm 7.0$ years. Six patients had diabetic nephropathy and 6 patients had history of previous cardiovascular surgery. Nine patients performed under emergency situation. The pathology of aortic lesions included 6 true aneurysms, 4 pseudoaneurysms, 4 acute dissections, 3 chronic dissections, and 2 severely calcified aortas.

\section{Results}

Average intubation duration and average ICU stay was 4.8 \pm 7.0 days, and $11.5 \pm 13.2$ days. Postoperative complications included pneumonias in 11 , which resulted in tracheostomy in 6 and reintubations in 7, gastro-intestinal complications in 4 and neurological complications in 3. There were 3 hospital deaths (14\%) including 1 early death. There were 7 late deaths including 3 aortic events

Cardiac Surgery, Nagoya University Graduate School of Medicine, Nagoya, Aichi, 466-8550, Japan 